\title{
Prevalence of C-shaped root canal in a Brazilian subpopulation: a cone-beam computed tomography analysis
}

\begin{abstract}
Daniela Brait Silva Ladeira(a) Adriana Dibo Cruz ${ }^{(b)}$ Deborah Queiroz Freitas(a) Solange Maria Almeida(a)
\end{abstract}

(a) Department of Oral Diagnosis, Piracicaba Dental School, Universidade Estadual de

Campinas - Unicamp, Piracicaba, SP, Brazil.

(b) Department of Specific Formation, Dental School, Universidade Federal Fluminense UFF, Nova Friburgo, RJ, Brazil.
Declaration of Interests: The authors certify that they have no commercial or associative interest that represents a conflict of interest in connection with the manuscript.

\section{Corresponding Author:}

Deborah Queiroz Freitas

E-mail: deborah@fop.unicamp.br

htrp://dx.doi.org/10.1590/S1806-83242013005000027 Epub Nov 25, 2013

Submitted: Jun 03, 2013

Accepted for publication: Sep 01, 2013

Last revision: Sep 17, 2013
Abstract: The aim of this study was to use cone-beam computed tomography $(\mathrm{CBCT})$ images to evaluate the prevalence and configurations of $\mathrm{C}$ shaped canals in permanent mandibular second molars among members of a Brazilian subpopulation. CBCT exams of 214 patients (406 teeth) were evaluated for: presence of C-shaped root canals, number of canals and direction of the root grooves (buccal or lingual). Of the 214 CBCT images examined, 192 showed intact bilateral molars, which were used to analyze the distribution of unilateral and bilateral occurrences of Cshaped canals. The prevalence of these canals was correlated with gender and age, and the number of canals was correlated with the direction of the root grooves using the chi-squared test $(\alpha=0.05)$. The prevalence of $\mathrm{C}$-shaped canals was $15.3 \%$. This prevalence did not differ with gender or age. Most of the C-shaped molars had three (43.5\%) or two (37.1\%) canals; $69.4 \%$ of the $\mathrm{C}$-shaped canals were grooved in the buccal direction; $68.3 \%$ of the $\mathrm{C}$-shaped cases were unilateral. In conclusion, there was a significant prevalence of $\mathrm{C}$-shaped canals in the mandibular second molars of the population studied; the C-shaped canal system varied in configuration, with a higher prevalence of three and two canals and unilateral occurrence; CBCT is a useful tool for endodontic diagnosis and treatment.

Descriptors: Anatomy; Cone-Beam Computed Tomography; Molar.

\section{Introduction}

Preoperative radiographs may aid in visualizing and observing the anatomy of variant canal systems, since recognition of such variations is an essential prerequisite for successful endodontic diagnosis and treatment. One of these variations is the C-shaped canal system, so named because of the axial plane morphology of the root canal, first documented in endodontic literature by Cooke and $\operatorname{Cox}^{1}$ in 1979. This variant is seen mostly in mandibular second molars, although it can also appear in maxillary and mandibular premolars and molars., ${ }^{2,3}$ The main anatomic feature of C-shaped canals is the presence of a fin or web connecting individual mesial and distal canals, which causes the canal axial section to have a C-shape, and which hinders thorough cleaning, shaping, and obturation. Therefore, recognition of a C-shaped canal configuration before treatment can facilitate more effective management of the root canal system. $^{3}$ 
Although traditional radiographs are very useful in the preoperative assessment of canal anatomy, they are unlikely to show the extent of complexities of the root canal system, because of the image superimposition provided by the two-dimensional images of conventional radiography.

Recently, cone-beam computed tomography (CBCT) was reported to be sufficiently precise to perform morphological analyses. ${ }^{4,5}$ Its advantages allow the clinician a more thorough understanding of the true morphology of root canal systems. ${ }^{6}$

The prevalence of a C-shaped canal in mandibular second molars has been estimated to range from $2.7 \%$ to $44.5 \%$, depending on the population and ethnic group:

- $2.7 \%-7.6 \%$ for American, ${ }^{1,7}$

- $8 \%$ for Turkish, ${ }^{8,9}$

- $10.6 \%$ for Saudi Arabian, ${ }^{10}$

- $19.1 \%$ for Lebanese, ${ }^{11}$

- $31.5 \%$ for Chinese ${ }^{12}$ and

- $32.7 \%-44.5 \%$ for Korean populations..$^{13,14}$

A study recently conducted in the Brazilian population evaluated the root canal configuration of mandibular molars, and showed that the prevalence of C-shaped canals in second molars was 3.5\%; however, the authors did not report the characteristics of its configuration, bearing in mind that the study was not limited to evaluating only the Cshaped canals. ${ }^{15}$ Therefore, the aim of this study was to use CBCT images to evaluate the prevalence and configurations of $\mathrm{C}$-shaped canals in the permanent mandibular second molars of members of a Brazilian subpopulation.

\section{Methodology} Patients and teeth

In this study, an evaluation was made of the mandibular second molars of patients who were referred to the Oral Radiologic Clinic at Piracicaba Dental School, Universidade Estadual de Campinas - Unicamp, Piracicaba, SP, Brazil, between February and November 2010, and who met the inclusion criteria described below, regardless of gender or age, thus characterizing a convenience sample. The exams were required for different treatment purposes not exclusive to this study. For the purpose of obtaining clear images of the mandibular second molars, teeth with physiological and/or pathological defects were excluded. Teeth were selected according to the following criteria:

- mandibular second molars with fully developed roots and with no periapical lesions;

- no root canal treatment;

- no root canals with open apices, resorption or calcification; and

- CBCT images of good quality, with field of view in which the mandibular second molars could be seen adequately.

A total of 214 patients met the inclusion criteria; 22 had a unilateral intact molar, and 192 had bilateral molars; a total of 406 teeth were analyzed. Bilateral molar data were used to analyze the distribution of unilateral and bilateral occurrences of C-shaped canals. The study group consisted of 130 females and 84 males, between 18 and 74 years of age (mean age of 29.9 years).

The study was approved by the Research Ethics Committee of the Universidade Estadual de Campinas - Unicamp (Protocol no. 53/2011) and a written consent of each patient was obtained.

\section{Radiographic techniques}

The CBCT images were performed using a Classic i-CAT scanner (Imaging Sciences International, Inc., Hatfield, USA), operating at $120 \mathrm{kVp}$ and $8 \mathrm{~mA}$, voxel $0.25 \mathrm{~mm}, 512 \times 512$ matrix, with no added filtration. All CBCT exposures were performed by an appropriately licensed radiologist.

\section{Images analysis}

The CBCT images were analyzed using XoranCat software version 3.1.62 (Xoran Technologies, Ann Arbor, USA). The contrast and brightness of the images were adjusted using the image processing tool in the software to ensure optimal visualization. The images were evaluated by two oral radiologists with at least one-year's experience in performing CBCT under dim light conditions. When a consensus could not be reached between the two examiners, an endodontist would assist in making the decision. 
Figure 1 - Sequence of axial images from the cervical third to the apical third of the root used to evaluate the presence of C-shaped canals. Images $\mathbf{A}$ and $\mathbf{B}$ show an example of a tooth considered as having a C-shaped canal, because it presents fused roots, a longitudinal groove on the buccal surface of the root, at least one axial section of the canal that shows the $C$ configuration and roots that are fused along the entire length, as indicated by the arrow. Images $\mathbf{C}$ and $\mathbf{D}$ show roots that are not fused from the middle third to the apical third of the root, in the case of a tooth without a C-shaped canal.
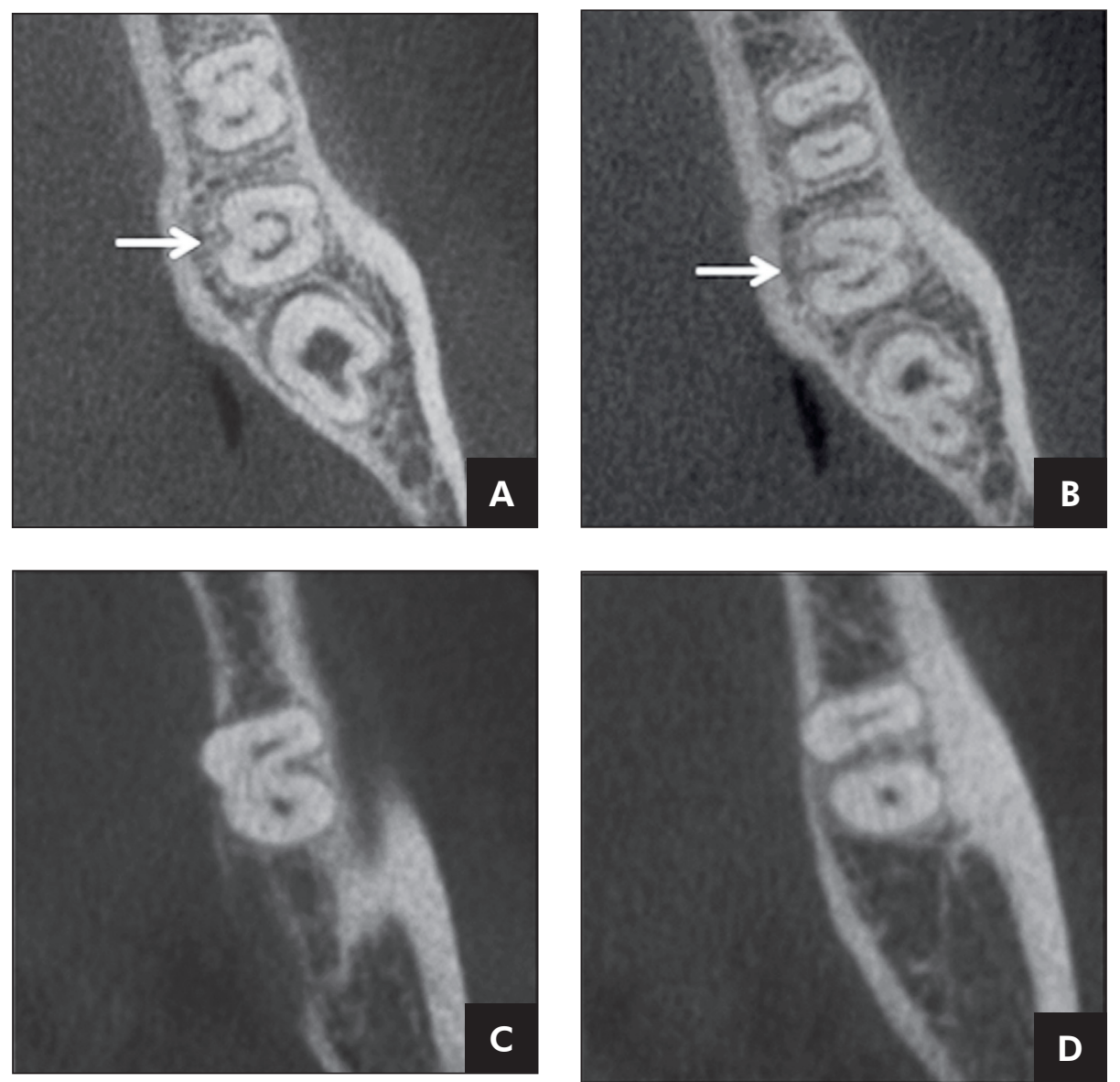

Determining of the C-shaped canal system in a mandibular second molar required that the tooth exhibit all of the following features adapted from those described by Fan et al.:16

I. fused roots,

II. a longitudinal groove on the lingual or buccal surface of the root,

III. at least one axial section of the canal showing a C configuration, and

IV. the roots fused along their entire length (Figure 1).

Accordingly, the images could be evaluated in axial, coronal and sagittal sections; however, to define the canals as C-shaped, the evaluators had to examine all axial sections of the roots. In assessing each eligible tooth, all axial sections of the roots were also used to evaluate the number of canals and the direction of the root grooves (buccal or lingual).

The frequency of the C-shaped canals was correlated with gender and age, and the number of the canals was correlated with the direction of the root grooves. These correlations were determined and assessed by the chi-squared test-used to determine whether there is a significant difference between the expected frequencies and the observed frequencies in studied variables-using the Stata Statistics/Data Analysis version 11.0 software (StataCorp., College Station, USA) with a significance level set at $5 \%$ $(\alpha=0.05)$.

\section{Results}

Of the 406 mandibular second molars examined in CBCT images, 62 (15.3\%) had a C-shaped root canal system. Considering the total 214 patients, $49(22.9 \%)$ showed a C-shaped canal. Of the 192 patients with bilateral permanent mandibular second molars, analyzed to determine the distribution of unilateral and bilateral C-shaped canals, 41 presented C-shaped canals, in that 28 patients $(68.3 \%$ of these cases) had a unilateral and 13 (31.7\%) had a 
bilateral C-shaped canal.

Table 1 shows the distribution of C-shaped canals in mandibular second molars by gender. No statistical difference was observed in this data. The distribution of $\mathrm{C}$-shaped canals in mandibular second molars by age is shown in Table 2. No statistical difference was observed in this data.

Table 3 shows the association between number of canals and the direction of the root grooves of teeth with a C-shaped root canal. Most of the Cshaped molars had three $(43.5 \%)$ or two $(37.1 \%) \mathrm{ca}-$ nals, mainly with root grooves in a buccal direction $(69.4 \% ; p<0.05)$, as illustrated in Figure 2.

\section{Discussion}

Many methods are used to investigate the root canal anatomy in vitro and in vivo. ${ }^{2}$ However, with the exception of radiographic exams, these methods either destroy the specimen by grinding or splitting, or preclude further use of the specimen, from procedures such as tooth clearing and dye assessment. An ideal technique is one that is accurate, simple, nondestructive, and most importantly, feasible in in vivo scenarios. ${ }^{4}$ Based on all of these factors, radiography has been opted for as the most practical and frequently used method to predict the root canal anatomy in both laboratory and clinical studies. ${ }^{3}$

However, it is difficult to detect the canal systems of mandibular molars with conventional radi- ography because the images of the roots overlap, especially in C-shaped roots, where the radiographic images may appear in diverse manners, depending on the exact nature and orientation of the root. The root may appear as a single, fused root or as two distinct roots with a communication. When it appears as two distinct roots with a communication, the C-shaped canal may not be easily recognized on conventional periapical films. ${ }^{17}$ CBCT makes this canal system more predictable, and is a good option clinically speaking, because it can detect Cshaped canal configurations. ${ }^{5,18}$ Today, some studies have been using micro-CT to investigate root canal morphology. ${ }^{3,19}$ This evaluation provides accurate data, but micro-CT cannot be used clinically yet. Therefore, this study used CBCT to evaluate the canal systems of 406 mandibular second molars in Brazilian individuals to identify the prevalence of C-

Table 1 - Frequency of C-shaped canals in mandibular second molars by gender [n(\%)].

\begin{tabular}{c|r|r|r|}
\hline \multirow{2}{*}{ C-shaped } & \multicolumn{2}{|c|}{ Gender } & \multirow{2}{*}{ Total } \\
\cline { 2 - 3 } & \multicolumn{1}{|c|}{ Female } & \multicolumn{1}{|c}{ Male } & \multicolumn{2}{|c}{} \\
\hline Absent & $205(50.5)$ & $139(34.2)$ & $344 \quad(84.7)$ \\
\hline Present & $43(10.6)$ & $19(4.7)$ & $62 \quad(15.3)$ \\
\hline Total & $248(61.1)$ & $158(38.9)$ & $406(100.0)$ \\
\hline
\end{tabular}

Fisher's exact test $(p>0.05)$.

Table 2 - Frequency of C-shaped canals in mandibular second molars by age $[\mathrm{n}(\%)]$.

\begin{tabular}{c|c|c|c|r|r|c}
\hline \multirow{2}{*}{ C-shaped } & \multicolumn{7}{|c|}{ Age (years) } & \multirow{2}{*}{ Total } \\
\cline { 2 - 8 } & $\leq 20$ & $21-30$ & $31-40$ & $41-50$ & $\geq 51$ & \\
\hline Absent & $50(12.3)$ & $190(46.8)$ & $64(15.8)$ & $16(3.9)$ & $24(5.9)$ & $344 \quad(84.7)$ \\
\hline Present & $6(1.5)$ & $29(7.1)$ & $15(3.7)$ & $8(2.0)$ & $4(1.0)$ & $62 \quad(15.3)$ \\
\hline Total & $56(13.8)$ & $219(53.9)$ & $79(19.5)$ & $24(5.9)$ & $28(6.9)$ & $406(100.0)$ \\
\hline
\end{tabular}

Likelihood ratio test $(p>0.05)$.

Table 3 - Correlation between number of canals and direction of root grooves [n(\%)].

\begin{tabular}{|c|c|c|c|c|c|}
\hline \multirow{2}{*}{$\begin{array}{l}\text { Direction of } \\
\text { root grooves }\end{array}$} & \multicolumn{4}{|c|}{ Number of canals } & \multirow{2}{*}{ Total } \\
\hline & 1 & 2 & 3 & 4 & \\
\hline Buccal & $2 \quad(3.2)$ & 18 (29.0) & $22(35.5)$ & $1(1.6)$ & $43 \quad(69.4)$ \\
\hline Lingual & $9(14.5)$ & $5 \quad(8.1)$ & $5 \quad(8.1)$ & $0(0.0)$ & 19 (30.6) \\
\hline Total & 11 (17.7) & $23(37.1)$ & 27 (43.5) & $1(1.6)$ & $62(100.0)$ \\
\hline
\end{tabular}

Likelihood ratio test $(p<0.05)$. 
Figure 2 - Examples of C-shaped groove directions. A: a case with a groove in the lingual direction; B: a case in the buccal direction.

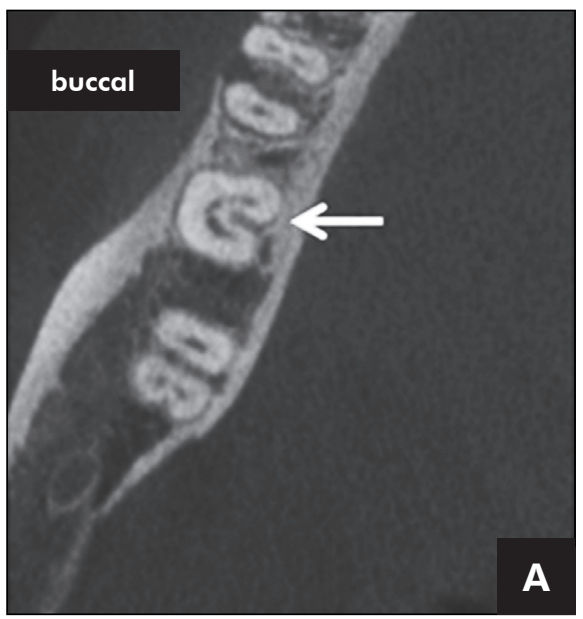

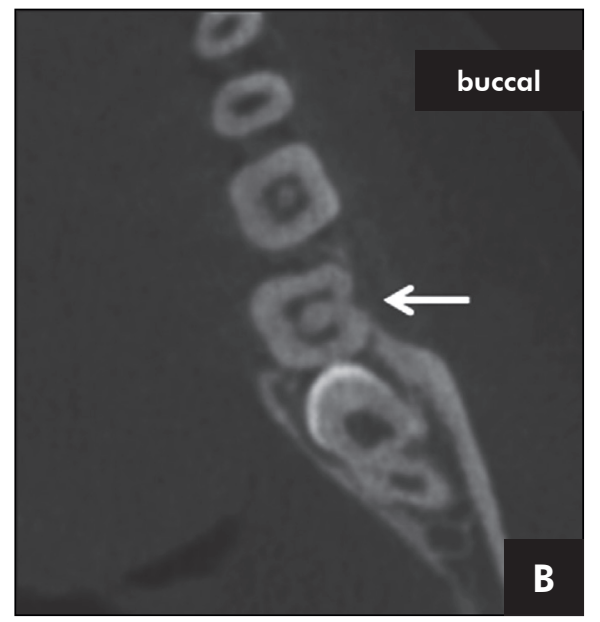

shaped canals.

In the literature, the C-shaped canal was most common in a Korean subpopulation, with a $31 \%-$ $45 \%$ prevalence. ${ }^{13,14,18}$ The Chinese population also presented high prevalence of C-shaped canals, as reported by Yang et al., ${ }^{12}$ Zheng et al. ${ }^{20}$ and Zhang et al. ${ }^{5}$ (32\%, 39\% and 29\%, respectively). C-shaped root and canal configurations were seldom found in Caucasian populations: Cooke and Cox, ${ }^{1}$ Weine $^{7}$ and Cimilli et al. ${ }^{8}$ reported a prevalence of $2.7 \%$, $7.6 \%$ and $8.1 \%$ of C-shaped canals, respectively. These data corroborate that canal shape was significantly related to race, with higher prevalence of C-shaped canals in Asians. This occurrence may explain the high number of studies in Asian populations and the lack of studies in others. To our knowledge, only one study evaluated the canal configurations in a Brazilian subpopulation; the authors found a $3.5 \%$ prevalence of C-shaped canals in second mandibular molars. ${ }^{15}$ The present investigation revealed a higher prevalence of C-shaped canals (15.3\%). However, it is difficult to make a direct comparison between both studies, since the authors did not indicate the features adopted to classify the root canal as C-shaped; moreover, they did not mention where the population studied was from. Even though these studies did not aim at gathering genetic-ethnic data, the fact that Brazil is a large country with extensive miscegenation could possibly explain the difference in results.

C-shaped canals present a complex and irregu- lar space containing potentially infected soft-tissue remnants or debris that may escape normal cleaning and filling procedures. ${ }^{21}$ Therefore, when Cshaped canals are identified, they may be debrided and obturated precisely to achieve successful root canal treatment. The access cavity and obturation for teeth with a C-shaped root canal system varies considerably and depends on the pulp morphology of the specific tooth. However, in general, alternative canal cleaning techniques, such as those using ultrasonics, would be more effective; an increased volume of irrigant and deeper penetration with small instruments using sonics or ultrasonics allows greater cleansibility in fan-shaped areas of the C-shaped canal. The obturation of C-shaped canals may require technique modifications: the mesial and distal canal spaces can be prepared and obturated as standard canals; however, sealing the buccal/lingual isthmus is difficult if lateral condensation is the only method used; therefore, application of thermoplasticized gutta-percha is more appropriate. ${ }^{21}$

In this study, no correlation was found between gender or age and the prevalence of C-shaped canals. These data are similar to the findings of the study by Zheng et al. ${ }^{20}$ Sert and Bayirli ${ }^{22}$ claimed that gender is an important factor to be considered in the preoperative evaluation of canal morphology for root canal treatment; however, the authors evaluated second premolars.

The C-shaped roots showed wide variations in their canal configuration, consistent with other 
reports. $., 5,8,14,20$ We found that $43.5 \%, 37.1 \%$ and $17.7 \%$ of the C-shaped molars had three, two and single canals, respectively. Only one second molar in the present study had four canals (1.6\%). Manning $^{23}$ also reported that C-shaped roots most frequently had three canals. However, our results are opposite from those of the study by Zhang et al., 5 which revealed that $57 \%, 29 \%$ and $14 \%$ of the Cshaped roots had a single canal, two canals and three canals, respectively. Both studies used the same tool for diagnosis: CBCT images. Therefore, the difference could not be attributed to the study method. We believe that the sample source may have caused this difference.

In the present study, most of the teeth with Cshaped canals were grooved in the buccal direction, which contrasts with the results by Helvacioglu-Yigit and Sinanoglu, ${ }^{9}$ Jin et al. ${ }^{14}$ and Zheng et al. ${ }^{20}$ However, these authors evaluated Turkish, Korean and Chinese populations, respectively. This could represent another anatomical variation related to ethnicity. The results also showed that there is a higher probability of the lingual direction in single C-shaped canal, whereas the buccal direction is more probable in C-shaped teeth with more than one canal. This data may be useful to guide instrumentation and filling, when a $3 \mathrm{D}$ image is not available.

Zheng et al. ${ }^{20}$ found that C-shaped canals were bilaterally distributed in $81 \%$ of the sample, whereas we found that bilateral C-shaped canals were less frequent $(31.7 \%)$. The fact that this anatomic variation is unilateral adds yet another diagnostic challenge, because when the method of exam provides less information to perform the diagnosis, it is com-

\section{References}

1. Cooke HG 3rd, Cox FL. C-shaped canal configurations in mandibular molars. J Am Dent Assoc. 1979 Nov;99(5):836-9.

2. Lu TY, Yang SF, Pai SF. Complicated root canal morphology of mandibular first premolar in a Chinese population using the cross section method. J Endod. 2006 Oct;32(10):932-6.

3. Fan B, Gao Y, Fan W, Gutmann JL. Identification of a Cshaped canal system in mandibular second molars-part II: the effect of bone image superimposition and intraradicular mon for the evaluator to use his previous knowledge and compare homologous anatomical structures to make his diagnosis. In any case, these data demonstrate that it is important for the professional to be aware of the likelihood that a C-shape canal may be present in the opposite molar, if a patient has a Cshaped canal in a mandibular second molar.

The importance of this study should be underscored, insofar as it is the first to evaluate the configurations of C-shaped canals in a sample of the Brazilian population; however, more studies are necessary, considering that a limitation of this study was that it was conducted on a local population. As indicated from the aforementioned studies, there is a strong relation between this type of anatomic variation and the characteristics of the population from which the sample was selected. ${ }^{2,8-15}$ We also encourage more studies to be conducted to determine the prevalence of anatomical variations in different ethnic groups, especially those that have not been evaluated previously.

\section{Conclusion}

There was a significant prevalence $(15.3 \%)$ of $\mathrm{C}$-shaped canals in the mandibular second molars in the population studied, with no predilection for gender or age. C-shaped canal systems varied considerably in regard to anatomical configuration, with a higher prevalence of three and two canals. CBCT can be a useful clinical tool for performing endodontic diagnosis and treatment when conventional radiographic views produce limited information and further radiographic details are required for endodontic diagnosis and treatment planning.

contrast medium on radiograph interpretation. J Endod. 2008 Feb;34(2):160-5.

4. Neelakantan P, Subbarao C, Ahuja R, Subbarao CV, Gutmann JL. Cone-beam computed tomography study of root and canal morphology of maxillary first and second molars in an Indian population. J Endod. 2010 Oct;36(10):1622-7.

5. Zhang R, Wang H, Tian YY, Yu X, Hu T, Dummer PM.

Use of cone-beam computed tomography to evaluate root and canal morphology of mandibular molars in Chinese individuals. Int Endod J. 2011 Nov;44(11):990-9. 
6. Wang Y, Zheng QH, Zhou XD, Tang L, Wang Q, Zheng $\mathrm{GN}$, et al. Evaluation of the root and canal morphology of mandibular first permanent molars in a western Chinese population by cone-beam computed tomography. J Endod. 2010;Nov;36(11):1786-9.

7. Weine FS. The C-shaped mandibular second molar: incidence and other considerations. Members of the Arizona Endodontic Association. J Endod. 1998 May;24(5):372-5.

8. Cimilli H, Cimilli T, Mumcu G, Kartal N, Wesselink P. Spiral computed tomographic demonstration of C-shaped canals in mandibular second molars. Dentomaxillofac Radiol. 2005 May;34(3):164-7.

9. Helvacioglu-Yigit D, Sinanoglu A. Use of cone-beam computed tomography to evaluate $\mathrm{C}$-shaped root canal systems in mandibular second molars in a Turkish subpopulation: a retrospective study. Int Endod J. 2013 Feb 27. DOI: 10.1111/iej.12094. Epub ahead of print.

10. Al-Fouzan KS. C-shaped root canals in mandibular second molars in a Saudi Arabian population. Int Endod J. 2002 Jun;35(6):499-504.

11. Haddad GY, Nehme WB, Ounsi HF. Diagnosis, classification, and frequency of C-shaped canals in mandibular second molars in the Lebanese population. J Endod. 1999 Apr;25(4):268-71.

12. Yang ZP, Yang SF, Lin YC, Shay JC, Chi CY. C-shaped root canals in mandibular second molars in a Chinese population. Endod Dent Traumatol. 1988 Aug;4(4):160-3.

13. Seo MS, Park DS. C-shaped root canals of mandibular second molars in a Korean population: clinical observation and in vitro analysis. Int Endod J. 2004;Feb;37(2):139-44.

14. Jin GC, Lee SJ, Roh BD. Anatomical study of C-shaped canals in mandibular second molars by analysis of computed tomography. J Endod. 2006 Jan;32(1):10-3.
15. Silva EJ, Nejaim Y, Silva AV, Haiter-Neto F, Cohenca N. Evaluation of Root Canal Configuration of Mandibular Molars in a Brazilian Population by Using Cone-beam Computed Tomography: An In vivo Study. J Endod. 2013 Jul;39(7):849-52.

16. Fan B, Cheung GS, Fan M, Gutmann JL, Bian Z. C-shaped canal system in mandibular second molars: Part I-Anatomical features. J Endod. 2004 Dec;30(12):899-903.

17. Huang RY, Cheng WC, Chen CJ, Lin CD, Lai TM, Shen EC, et al. Three-dimensional analysis of the root morphology of mandibular first molars with distolingual roots. Int Endod J. 2010 Jun;43(6):478-84.

18. Seo DG, Gu Y, Yi YA, Lee SJ, Jeong JS, Lee Y, et al. A biometric study of C-shaped root canal systems in mandibular second molars using cone-beam computed tomography. Int Endod J. 2012 Sep;45(9):807-14.

19. Liu N, Li X, Liu N, Ye L, An J, Nie X, et al. A micro-computed tomography study of the root canal morphology of the mandibular first premolar in a population from southwestern China. Clin Oral Investig. 2013 Apr;17(3):9991007.

20. Zheng Q, Zhang L, Zhou X, Wang Q, Wang Y, Tang L, et al. C-shaped root canal system in mandibular second molars in a Chinese population evaluated by cone-beam computed tomography. Int Endod J. 2011 Sep;44(9):857-62.

21. Jafarzadeh H, Wu YN. The C-shaped root canal configuration: a review. J Endod. 2007 May;33(5):517-23.

22. Sert S, Bayirli GS. Evaluation of the root canal configurations of the mandibular and maxillary permanent teeth by gender in the Turkish population. J Endod. 2004;Jun;30(6):391-8.

23. Manning SA. Root canal anatomy of mandibular second molars. Part II. C-shaped canals. Int Endod J. 1990 Jan;23(1):40-5. 